\title{
P03-1-37 Poster session
}

\section{Ubiquitination of EAAT2 is involved in the impairment of stress adaptation}

\author{
Hiroko Miyagishi, Minoru Tsuji, Atsumi Saito, Kazuhiro Kurokawa, Kazuya Miyagawa, \\ Hiroshi Takeda
}

Department of Pharmacology, School of Pharmacy, International University of Health and Welfare, Japan

The ability to adapt to stress is an important defensive function of a living body, and impairment of this ability may contribute to some stress-related disorders. Previously, we reported that mice exposed to repeat excessive restraint stress (240 min restraint stress once a day for 14 days) showed emotional abnormality, which suggests an inability to adapt to stressful conditions. Moreover, the protein level of excitatory amino acid transporter-2 (EAAT2) was decreased in the hippocampus of mice that had been exposed to inadaptable stress, while there was no change in prefrontal cortex. Recently, EAAT2 has been reported to be ubiquitinated by E3 ubiquitin ligase neural precursor cell expressed developmentally downregulated protein 4-2 (Nedd4-2). In order to elucidate the role of EAAT2 in stress-maladaptation, the present study investigated whether hippocampal ubiquitination of EAAT2 and expression of Nedd4-2 are facilitated by exposed to inadaptable stress in mice.

Mice were repeatedly exposed to inadaptable stress, i.e. restraint stress for $240 \mathrm{~min} /$ day for 14 days. After the final exposure to stress, brains of mice were rapidly removed and the hippocampus and prefrontal cortex were dissected. Proteins were extracted from brain tissues. Ubiquitination of EAAT2 and expression of Nedd4-2 was assessed by immunoprecipitation and western blotting, respectively.

Immunoprecipitation revealed that the level of ubiquitination of EAAT2 was increased in the hippocampus of stressmaladaptive mice. In the western blot analysis, increase in the hippocampal level of Nedd4-2 was also found in stressmaladaptive mice. However, no change in the level of expression of Nedd4-2 was observed in the prefrontal cortex of stress-maladaptive mice.

The present study demonstrated that, in the hippocampus of stress-maladaptive mice, ubiquitination of EAAT2 is enhanced accompanying an increase in the expression of Nedd4-2. Therefore, it is suggested that the enhancement of degradation of EAAT2 promoted by Nedd4-2-mediated ubiquitination is involved in the impairment of stress adaptation. 